\title{
Die Pflege im Managementarrangement
}

Martin Feisst

Im Rahmen einer dreijährigen DFG-geförderten Studie mit dem Titel „Entscheidungsfindung im Krankenhausmanagement“ wurden 14 Klinikleitungen einer intensiven Betrachtung unterzogen. Dabei wurde deutlich, dass eine gelungene Managementpraxis maßgeblich davon abhängt, zu welchem spezifischen Arrangement die Pflegedirektionen mit den Ärztlichen- und kaufmännischen Leitungen in der Praxis zusammenfinden.

» Die bundesdeutschen Krankenhäuser stehen unter enormem finanziellem Druck. Dies ist bereits vielfältig diskutiert worden und braucht entsprechend nicht mehr explizit ausgeführt werden (Simon 2016, Bode \& Vogd 2016). Bekanntermaßen haben in den letzten zehn Jahren 30-40\% der Krankenhäuser rote Zahlen geschrieben (vgl. Blum et. al 2014 \& 2015). Wie sich auch aus Sicht der von uns befragten Klinikleitungen gezeigt hat, ist weitgehend unstrittig, dass gerade in den Ballungszentren ein Überangebot an Krankenhäusern besteht. Allerdings werden von Seiten der Politik keine gezielten Schließungen vorgenommen; vielmehr wird abgewartet, welche Krankenhäuser dem finanziellen Druck standhalten und welche nicht. Unter diesen Bedingungen kommt dem Krankenhausmanagement eine wichtige Rolle zu. Denn Aufgabe eines jeden Managements ist es, nicht zuletzt aufgrund der Verantwortung für die Mitarbeiter, das Überleben des eigenen Hauses zu sichern.

Während sich viele Geschäftsführungen und Kaufmännische Leitungen dem Vorwurf ausgesetzt sehen, Gewinne auf Kosten der Patientenbehandlung generieren zu wollen, wird in unserer Studie eine weitere und zusätzliche Perspektive deutlich: Es gehe nicht um Gewinne, vielmehr müssen die Verluste kompensiert werden, die aus einer systematischen Unterfinanzierung entstehen. Bleiben in dem dualen Finanzierungsmodell die Investitionszahlungen aus oder sind zu gering, müssen unter anderem bauliche Sanierungsmaßnahmen durch erwirtschaftete Überschüsse aus der DRGVergütung bestritten werden (Vogd et. al 2017, 215 \& 348). Es wird deutlich,

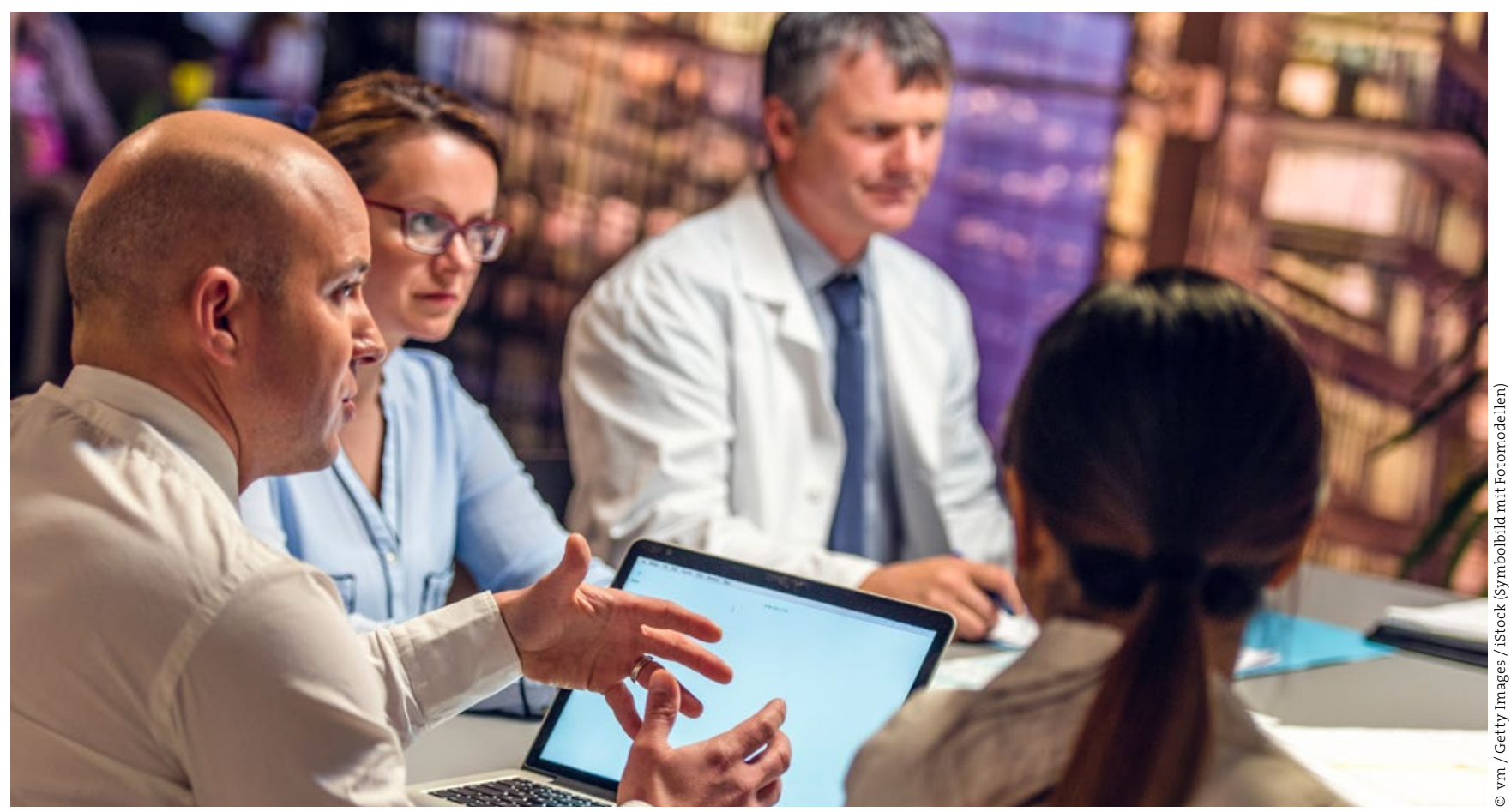

Abb. 1: Optimal ist es, wenn alle am Tisch gehört werden. 
dass sich jede Berufsgruppe ihren eigenen Spannungen ausgesetzt sieht. So muss auch die Pflege im Management trotz der prekären wirtschaftlichen Lage ihre Interessen positionieren.

\section{Der Wunsch: Eine spannungsfreie Organisation}

Besonders im Management tritt das Spannungsfeld zwischen Selbsterhalt und öffentlicher Daseinsvorsorge offen zu Tage. In der Folge kommt es zu einer enormen Verdichtung der Arbeitsprozesse auf allen Ebenen (Braun 2014, Ethikrat 2016). Durch die Verkürzung der Liegezeiten und finanzielle Bevorzugung schwerer Fälle durch das DRGSystem muss immer mehr in immer kürzerer Zeit geleistet werden. Anforderungen der Patientenbehandlung müssen mit betriebswirtschaftlichen Erfordernissen arrangiert werden. Dabei ist die Frage, was betriebswirtschaftlich sinnvoll und was medizinisch notwendig ist, nicht immer eindeutig zu klären. Und auch zwischen ärztlichen und pflegerischen Denkweisen kommt es zu Spannungen.

Während die Pflege ein ganzheitliches, umfassendes Denken für sich reklamiert (sowohl mit Blick auf den $\mathrm{Pa}$ tienten als auch auf die damit verbundenen Organisationsprozesse), tritt die Ärzteschaft punktuell in Erscheinung (sowohl mit Blick auf den Patienten als auch auf die Organisationsprozesse). All dies ist im Grundsatz nichts Neues, wie bereits Jürgen Rhode in seiner Studie zur „Soziologie des Krankenhauses“ (1974) festgestellt hat. Er verwies darauf, dass es zwar die ,naive Sehnsucht' (ebd. 359) nach der spannungsfreien Organisation gibt, dies aber schon immer mehr Wunsch als Wirklichkeit war. Das bedeutet wiederum nicht, dass jede Art von Spannung stoisch ertragen werden muss und kein Handlungsbedarf angezeigt ist. Die Frage ist vielmehr, ab wann der sprichwörtliche Bogen überspannt ist. Gerade das Management der Krankenhäuser sieht sich unter den derzeitigen Bedingungen des Gesundheitswesens einer Vielzahl unterschiedlichster Spannungen ausgesetzt. Ihre Aufgabe liegt darin, diese aufzugreifen, auszuhalten und zu moderieren und auch unter finanziellem Druck sowohl eine medizinisch vertretbare Patientenbehand- lung als auch akzeptable Arbeitsbedingungen $\mathrm{zu}$ realisieren.

\section{Von der Managerin zum Managementarrangement}

Wir gehen weiterhin davon aus, dass das Gelingen einer verantwortlichen Managementpraxis nicht der heroischen Leistung eines einzelnen Managers zu verdanken ist; vielmehr ist in den Blick zu nehmen, welche Arrangements sich in der Praxis ausbilden. Hier spielen neben den formalen Stellen und somit der Hierarchie auch die konkreten Personen sowie die Rahmenbedingungen eine entscheidende Rolle. Entsprechend greift es zu kurz, die eine Managerin zu identifizieren. Vielmehr ist der Blick auf eine spezifische Praxis der Krankenhausleitung zu richten, die sich aus dem Zusammenspiel der ärztlichen und kaufmännischen Leitung sowie der Pflegedirektion (und ggf. weiteren relevanten Managementakteuren) ergibt.
Bei Krankenhäusern handelt es sich zudem um professionelle Bürokratien (Mintzberg). Die medizinische Behandlung folgt einer starken professionellen Eigenlogik. Ärztliches wie pflegerisches Handeln weisen (je) eigene Wertprämissen auf, die sich maßgeblich von betriebswirtschaftlichen Wertvorstellungen unterscheiden. Das bedeutet zwar nicht, dass sich diese zwangsweise ausschließen oder gegenläufig sein müssen. Allerdings ist dies einer der Gründe, warum sich Chefärzte (und aufgrund der Professionalisierung zunehmend auch die Pflegedirektionen) nur bedingt betriebswirtschaftlich, steuern' lassen sehr zum Leidwesen vieler Geschäftsführungen. Eben deshalb müssen die unterschiedlichen Logiken und Werte im Management zueinander in Beziehung gesetzt werden und - vermittelt durch die jeweiligen ärztlichen, pflegerischen und kaufmännischen Leitungen - zu einem Arrangement finden.

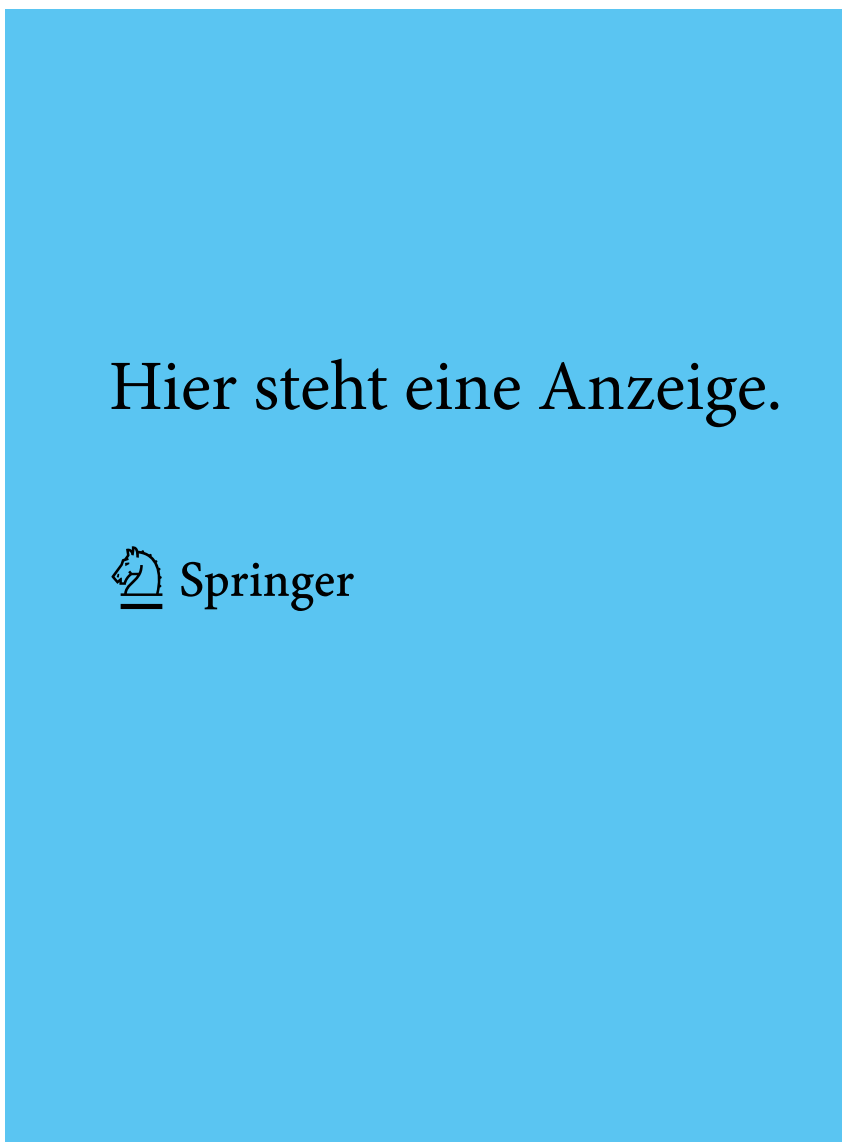




\section{Die Rolle der Pflege in den verschiedenen Arrangements}

Obschon sich jedes Krankenhausmanagement aufgrund der angespannten Situation des Gesundheitssystems mit demselben Problem konfrontiert sieht, ist damit noch nicht entschieden, wie im Einzelfall damit umgegangen wird. Doch die angeführten professionellen Eigenlogiken können nur dann im Sinne erfolgreicher Patientenbehandlung wirksam werden, wenn ihnen entsprechende Spielräume ermöglicht werden (Oevermann 1990). Ansonsten kommt es zu den beobachtbaren Deprofessionalisierungstendenzen.

Welchen Einfluss Pflegedirektionen im Krankenhausmanagement ausüben, hängt dabei oft auch von situativen Faktoren und der Gesamtkonstellation der Klinikleitungen ab. Hier lassen sich verschiedene Arrangements rekonstruieren, die in unterschiedlichen Spielarten immer wieder anzutreffen sind (vgl. Vogd et. al 2017, S. 219 ff).

Im Folgenden werden drei von uns rekonstruierte Arrangements kurz skizziert.

\section{Fall 1: Betriebswirtschaftliche} Logik übernehmen

Am Beispiel eines Krankenhauses in privater Trägerschaft eines bundesweit agierenden Großkonzerns lässt sich mit Blick auf die Pflegedirektionen ein sehr typisches Arrangement beobachten: Im konkreten Fall haben wir es mit einer klassischen Trias von Ärztlichem Direktor, Pflegedirektorin und Verwaltungsleiter zu tun. Der Verwaltungsleiter, ein Kaufmann, steht der Trias zugleich auch als leitender Geschäftsführer vor. Dieser vermag es, die betriebswirtschaftliche Logik als handlungsleitende Entscheidungsprämisse zu etablieren, nach der sich alles zu richten hat. Das gelingt ihm unter anderem deshalb, weil durch den Ärztlichen Direktor keine eigenständige Position eingenommen wird, die gleichwertig zu den wirtschaftlichen Anforderungen artikuliert wird. Den Chefärzten und ihrem Sprecher kommt tendenziell nur noch die Rolle zu, Widerstand gegen Zumutungen zu artikulieren, die dem ärztlichen Ethos in inakzeptabler Weise zuwiderlaufen.
Die Pflegedirektorin leistet in diesem Arrangement noch nicht einmal das. Zwar fühlt sie sich in der eigenen Darstellung auf Augenhöhe, da ihr der Geschäftsführer alles bereitwillig erklärt und sie ihre Meinung sagen darf. In der eingehenden Analyse der Interviews zeigt sich jedoch deutlich, wie sich die Pflegedirektorin dem Geschäftsführer und dessen (sehr erfolgreichen) betriebswirtschaftlichen Denkweise unterordnet, indem sie diese (unbewusst) übernimmt und keine Gegenposition einnimmt. Die prekäre Situation der Pflege im eigenen Haus findet so nicht einmal Erwähnung - von dieser erfahren wir erst durch den Chefarzt für Innere Medizin.

Wichtig zu erwähnen ist, dass sich dieses Arrangement nicht der privaten Trägerschaft zurechnen lässt, sondern auch in Fällen freigemeinnütziger Träger beobachtet werden konnte (Vogd et. al 2017, S. 121 ff.).

\section{Fall 2: Autonomiespielräume trotz steiler Hierarchie}

In einem anderen Klinikum vergleichbarer Bettenzahl und gleicher Trägerschaft zeigte sich, dass trotz einer wesentlich steileren Hierarchie die Position der Pflege deutlich mehr Eingang in die Entscheidungsprozesse findet als im Fall zuvor. Obschon auch hier die Personaldecke dünn und die Belastung hoch ist (die Pflegedirektorin springt in Extremfälle selbst auf Station ein), darf sie in diesem Arrangement nicht nur ihre Meinung sagen - ihre Position wird ernstgenommen, sie wird gehört. Und das, obwohl alle Entscheidungen letztlich durch den kaufmännischen Leiter getroffen werden. Auch der Ärztliche Direktor hat in diesem Haus formal-hierarchisch lediglich beratende Funktion und weiß, dass die Entscheidungen in der Praxis an anderer Stelle getroffen werden.

Nichtsdestotrotz finden wir hier einen kaufmännischen Leiter vor, der zwar die Entscheidungen allein oder auch mit seinem Vorgesetzten des Trägers trifft, hierfür aber die Position der Pflege und der Ärzteschaft auf eine Weise ernst nimmt, dass diese sich gut vertreten fühlen. Ein Chefarzt bringt dies auf die Formel: „Wir dürfen unsere Meinung sagen, aber wir werden auch gehört". Umgekehrt verzichtet der Kaufmännische Leiter auf ein zu strenges Controlling, so dass Ärzteschaft und Pflege - bei aller Anhaltung zur Wirtschaftlichkeit - nicht jede Einzelentscheidung betriebswirtschaftlich prüfen können oder müssen. Er behält sich nach eigener Aussage das Gespür für die Zahlen und die betriebswirtschaftlichen Prozesse vor und vertraut in medizinischen Fragen umgekehrt den Ärzten und deren Expertise. Die Ärzte, so der kaufmännische Leiter, können die Zahlen gar nicht richtig einordnen und würden die falsche Schlüsse ziehen, wenn beispielsweise ein großes Minus oder Plus auf ihrer Station zu verzeichnen sind. Damit kommt es zu einem Arrangement, in dem trotz der auch hier auftretenden, üblichen Spannungen zwischen Ärzteschaft, Pflege und Verwaltung und trotz der formalhierarchischen Ungleichberechtigungen jeder Profession - auch der Pflege - die jeweilige Expertise und entsprechende Autonomiespielräume zugestanden werden.

Bedingung hierfür ist neben der Leistungsbereitschaft der Mitarbeiter - gerade diese scheint in diesem Haus unabdingbar - ein kaufmännischer Leiter, der nicht nur betriebswirtschaftlich denken kann, sondern auch ärztlichen und pflegerischen Perspektiven einen Wert beimisst.

\section{Fall 3: Aushandlung auf Augenhöhe}

Abschließend sei noch auf das Arrangement eines Krankenhauses in katholischer Trägerschaft verwiesen. Rein formal finden wir in diesem Haus, welches deutlich größer ist, als die beiden zuvor genannten, eine Geschäftsführung aus einem Vorsitzenden der Geschäftsführung (Kaufmann) und einem hauptamtlichen Ärztlichen Direktor. Gemeinsam mit dem Verwaltungsleiter und der Pflegedirektorin bilden sie zu viert die Klinikleitung. Obschon diese vier nicht alle über dieselben Befugnisse verfügen (der Vorsitzende der Geschäftsführung und der Ärztliche Direktor sind letztentscheidungsbefugt), finden wir in diesem Krankenhaus unserer Analyse zufolge eine sehr gleichberechtigte Klinikleitung vor. Alle wichtigen Entscheidungen werden abgestimmt und 
gemeinsam gefällt. Das bedeutet nicht, dass hier niemand engagiert für die eigenen Belange eintritt. Via Abstimmung wird jedoch die gemeinsame Linie ermittelt und nach außen vertreten. Die Pflegedirektorin konnte so zuletzt einen deutlichen Stellenzuwachs in ihrem Bereich verzeichnen.

Ein solcher Modus ist allerdings hoch voraussetzungsvoll: Nach Aussage der Pflegedirektorin reiche es eben nicht, sich ,stur hinzustellen' und ,ich will aber' zu sagen. Sie muss eine Personalaufstockung gut begründen und entsprechend in der Leitungsrunde vorbringen. Das bedeutet, dass sie auch in der Lage sein muss, die Denkweise der anderen Klinikleiter verstehen und nachvollziehen zu können und auch an anderer Stelle Zugeständnisse zu machen. Dass dieses Management einen so erfolgreichen Modus gefunden hat, liegt offensichtlich nicht nur an der formal-hierarchischen Aufstellung (selbst hier könnte die Pflege prinzipiell ganz einfach übergangen werden). Entscheidend ist vielmehr, dass jeder der vier Klinikleiter es versteht, seine eigenen Belange einzubringen, dabei aber nicht übersieht, dass die anderen dies mit der gleichen Berechtigung ebenfalls tun.

\section{Fazit: Anerkennung der unterschiedlichen Positionen}

Das entscheidende Moment liegt also nicht so sehr in einer formalen Gleichstellung aller Beteiligten - auch wenn dies gerade auch in der Symbolwirkung nicht zu unterschätzen ist. Ent- scheidend ist vielmehr das Ausmaß, in dem die Akteure nicht nur willig, sondern auch fähig sind, die jeweils anderen Positionen und Logiken mitzudenken. Das klingt trivial, ist aber in der Praxis nach unseren Erkenntnissen eine der Grundvoraussetzungen für ein erfolgreiches Management. Das verdeutlicht, dass es nicht ausreicht, dass es den einen ,klugen' Manager gibt. Es kommt eben vielmehr auf das Zusammenspiel, auf das Arrangement aller Beteiligten an. Es bedarf hier sowohl Pflegedirektionen, die betriebswirtschaftlich denken können, ohne aber dabei ihre eigene Position aufzugeben (eine ernsthafte Gefahr, wie wir beobachten konnten) als auch Betriebswirte, die den Wert ärztlicher und pflegerischer Perspektiven anerkennen und verstehen. Kurzum: Es reicht nicht, dass jeder seine Meinung sagen darf, die jeweils anderen müssen auch willig und befähigt sein, diese zu hören und ernstnehmen.

Hierbei macht die Trägerschaft nach unseren Erkenntnissen nicht den entscheidenden Unterschied, zumal die Rechtsform zwischen öffentlicher, freigemeinnütziger und privater Trägerschaft in den meisten Fällen weitestgehend vergleichbar ist (Busse et al. 2010, S. 50). Und ohne eine straffe betriebswirtschaftliche Organisation ist es unter den derzeitigen Bedingungen des Gesundheitssystems ohnehin nicht möglich, einen im medizinischen Sinne erfolgreichen Krankenhausbetrieb aufrecht zu erhalten. Die entscheidende Frage der Ökonomisierung der Klinik lautet dann: Kommen

\section{Zusammenfassung}

Durch den enormen finanziellen Druck stehen die Krankenhäuser im Spannungsfeld zwischen Selbsterhalt und öffentlicher Daseinsvorsorge. Besonders den Krankenhausleitungen kommt die Aufgabe zu, mit den auftretenden Spannungen in einer Weise umzugehen, dass dennoch erträgliche Arbeitsbedingungen herrschen und eine vertretbare medizinische Patientenbehandlung möglich ist. Ob und wie sie dies schaffen, hängt maßgeblich davon ab, zu welchem Arrangement pflegerischer, ärztlicher und betriebswirtschaftlicher Interessen gefunden wird und ob der Pflege wie der Ärzteschaft entsprechende Handlungsspielräume erwirtschaftet werden können. Dies hängt weder mit der formalhierarchischen Gleichstellung aller Berufsgruppen noch mit der Trägerschaft zusammen. Entscheidend ist vielmehr, inwiefern alle Beteiligten zu einem Arrangement zusammenfinden, in der alle berufsspezifischen Eigenlogiken zur Geltung kommen.

Schlüsselwörter: Management, Krankenhaus, Pflege, Ökonomisierung, Managementarrangements, Qualitative Studie

die hierdurch gewonnenen Freiräume den Ärzten und Pflegeden zugute (Fall 2) oder der wirtschaftlichen Expansion des Konzerns (Fall 1)? Mit Blick auf die Entwicklungen des Gesundheitssystems bleibt fraglich, ob es zukünftig selbst dem besten Management noch gelingen kann, solche Spielräume zu erwirtschaften.

\section{Literatur}

Blum, K., Löffert, S., Offermanns, M., \& Steffens, P. (2014). Krankenhaus-Barometer. Umfrage 2014. Düsseldorf: Deutsches Krankenhaus Institut.

Blum, K., Löffert, S., Offermanns, M., \& Steffens, P. (2015). Krankenhaus-Barometer. Umfrage 2015. Düsseldorf: Deutsches Krankenhaus Institut.

Bode, I./Vogd, W. (2016): Einleitung. Mutationen des Krankenhauses. In: dies. (Hrsg.): Mutationen des Krankenhauses. Soziologische Diagnosen in organisations- und gesellschaftstheoretischer Perspektive. Wiesbaden, S. 1-25.

Braun, B. (2014): Auswirkungen der DRGs auf Versorgungsqualität und Arbeitsbedingungen im Krankenhaus. S. 91-114 in: Manzei, A./

Schmiede, R. (Hrsg.), Theoretische und empirische Analysen zur Ökonomisierung von Medizin und Pflege. Wiesbaden: Springer VS.

Busse, R., Schreyögg, J., \& Tiemann, O. H. (2010). Management im Gesundheitswesen. Berlin, Heidelberg, New York: Springer.

Ethikrat, D. (2016). Patientenwohl als ethischer Maßstab für das Krankenhaus. Stellungnahme 5. April 2016. Berlin: Deutscher Ethikrat.

Oevermann, U. (1990). Klinische Soziologie. Konzeptualisierung, Begründung, Berufspraxis und Ausbildung. Frankfurt Main.

Rohde, J.(1974): Soziologie des Krankenhauses. Zur Einführung in die Soziologie der Medizin. Stuttgart.

Simon, M. (2016): Die ökonomischen und strukturellen Veränderungen des Krankenhausbereichs seit den 1970er Jahren. In: Bode, I./ Vogd, W. (Hrsg.): Mutationen des Krankenhauses. Soziologische Diagnosen in organisations- und gesellschaftstheoretischer Perspektive. Wiesbaden, S. 29-46

Vogd, W./Feißt, M./Molzberger, K./Ostermann, A./Slotta, J. (2017): Entscheidungsfindung im Krankenhausmanagement. Zwischen gesellschaftlichem Anspruch, ökonomischen Kalkülen und professionellen Rationalitäten. Wiesbaden.

Autorenkontakt:

Martin Feißt ist wissenschaftlicher Mitarbeiter am Lehrstuhl für Soziologie an der Universität Witten/Herdecke. Seine Arbeitsschwerpunkte liegen im Bereich der Organisationsforschung sowie der rekonstruktiven Sozialforschung.

E-Mail: martin.feisst @uni-wh.de 